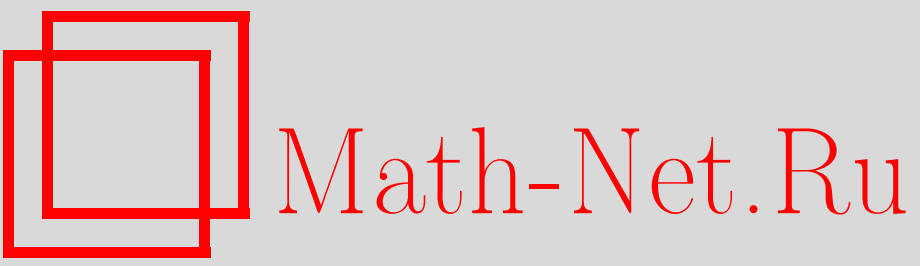

Р. В. Плыкин, О структуре централизаторов аносовских диффеоморфизмов тора, УМH, 1998, том 53, выпуск 6, 259-260

DOI: https://doi.org/10.4213/rm104

Использование Общероссийского математического портала Math-Net.Ru подразумевает, что вы прочитали и согласны с пользовательским соглашением

http://www.mathnet.ru/rus/agreement

Параметры загрузки:

IP: 52.23 .180 .231

26 апреля 2023 г., 16:06:16

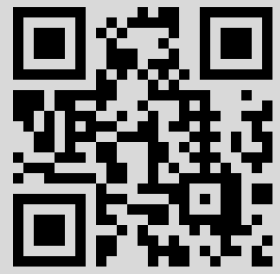




\title{
О СТРУКТУРЕ ЦЕНТРАЛИЗАТОРОВ АНОСОВСКИХ ДИФФЕОМОРФИЗМОВ ТОРА
}

\author{
Р. В. Плыкин
}

Пусть $\operatorname{Diff}(M)$ - группа $C^{\infty}$-диффеоморфизмов гладкого, связного, компактного многообразия $M$, наделенная $C^{\infty}$-топологией. Централизатором $Z(f)$ диффеоморфизма $f \in \operatorname{Diff}(M)$ называется подмножество $\operatorname{Diff}(M)$, состоящее из диффеоморфизмов, коммутирующих с $f$, которое, как нетрудно видеть, также образует группу.

Основным результатом этой заметки является следующая теорема, которая вместе с приводимыми ниже примерами дает отрицательный ответ на вопрос С. Смейла [1], [2]: может ли диффеоморфизм компактного многообразия $M$ быть приближен в $C^{r}$-топологии при $r \geqslant 1$ диффеоморфизмами, которые коммутируют лишь со своими итерациями?

Теорема. Пусть $A$ - аносовский диффеоморфизм n-тора, определяемьй унимодулярной матричей, обладающей неприводимым характеристическим многочленом и спектром $\operatorname{spec} A=\left\{\lambda_{1}(A), \lambda_{2}(A), \ldots, \lambda_{k}(A), \mu_{1}(A), \mu_{1}(\bar{A}), \ldots, \mu_{s}(A), \mu_{s}(\bar{A})\right\}$, где $\lambda_{i}(A)$ - вещественные, а $\mu_{j}(A)$ - комплексные характеристические числа. Тогда чентрализатор $Z(A)$ изоморфен группе $Z^{l} \oplus F$, где $1 \leqslant l \leqslant k+s-1, F-$ конечная коммутативная группа ( $s=0$ - случай вещественного спектра, $k=0$ - случай комплексного спектра).

Заметим, что вследствие леммы Гаусса [3] условия о неприводимости характеристического многочлена выполнены для всех матриц, спектр которых разделен единичной окружностью комплексной плоскости на два множества, в одном из которых содержится единственный элемент.

Доказательство теоремы использует следующие леммы.

Лемма 1. Если $B$ - автоморфизм тора, принадлежащий чентрализатору $Z(A)$, где $А$ удовлетворяет условиям теоремь, то $A$ и $B$ имеют одинаковый набор собственных векторов, задающих базис в комплексном пространстве $C^{n}$, и обратно, если автоморфизм $B$ имеет хотя бы один общий с $A$ собственный вектор, то $B \in Z(A)$.

Доказательство леммы вытекает из того, что если $e_{1}, e_{2}, \ldots, e_{k}, f_{1}, \bar{f}_{1}, \ldots, f_{s}, \bar{f}_{s}$ - базис, составленньй из собственных векторов автоморфизма $A$, то $B e_{1}, B e_{2}, \ldots, B e_{k}, B f_{1}, B \bar{f}_{1}, \ldots$, $B f_{s}, B \bar{f}_{s}$ - также базис автоморфизма $A$ с теми же собственными значениями.

Можно убедиться также в том, что координаты любого собственного вектора $e$ автоморфизма $A$ являются рационально независимыми алгебраическими числами. Поэтому в случае $A B \neq B A$ соотношение $(A B-B A) e=0$ порождает нетривиальную рациональную зависимость между координатами $e$, что невозможно.

Из последнего рассуждения легко вьводится следующая лемма.

Лемма 2. Если автоморфизмы тора $B_{1}, B_{2} \in Z(A)$ имеют одинаковые собственные числа при одном из собственных векторов, то $B_{1}=B_{2}$, если же әти собственные числа отличаются знаком, то $B_{1}=-B_{2}$.

Для доказательства теоремы заметим, что согласно лемме 2 спектр $\left(\lambda_{1}(B), \ldots, \lambda_{k}(B)\right.$, $\left.\rho_{1}(B) e^{i 2 \pi \varphi_{1}(B)}, \rho_{1}(B) e^{-i 2 \pi \varphi_{1}(B)}, \ldots, \rho_{s}(B) e^{i 2 \pi \varphi_{s}(B)}, \rho_{s}(B) e^{-i 2 \pi \varphi_{s}(B)}\right)$ автоморфизма $B \in Z(A)$ восстанавливает $B$, при этом перемножению двух автоморфизмов соответствует почленное перемножение их спектров.

Спектры образуют подгруппу $\operatorname{Spec} Z(A)$ группы $R_{*}^{k} \oplus C_{*}^{s}$, где $R_{*}\left(C_{*}\right)$ - мультипликативная группа вещественных (комплексных) чисел.

Как подгруппа топологической группы $\operatorname{Spec} Z(A)$ дискретна, в чем убеждаемся, применяя теорему Виета к сходящейся последователшности точек $\operatorname{Spec} Z(A)$, и замечаем вследствие целочисленности коэффициентов характеристических полиномов, соответствующих точкам этой последовательности, что различных полиномов конечное множество вопреки бесконечности выбранной последовательности. 
Заметим также, что более короткие последовательности $\left\{\lambda_{1}, \ldots, \lambda_{k-1}, \rho_{1}, \ldots, \rho_{s}, e^{i 2 \pi \varphi_{1}}\right.$, $\left.\ldots, e^{i 2 \pi \varphi_{s}}\right\}$ в случае $k \geqslant 1$ (или $\left\{\rho_{1}, \ldots, \rho_{s-1}, e^{i 2 \pi \varphi_{1}}, \ldots, e^{i 2 \pi \varphi_{s}}\right\}$, если $k=0$ ) также образуют дискретную подгруппу группы $R_{*}^{k-1} \oplus R_{+}^{s} \oplus T^{s}$ (соответственно $R_{+}^{s-1} \oplus T^{s}$ ), где $R_{+}-$мультипликативная группа положительных вещественных чисел, $T$ - мультипликативная группа комплексных чисел, равных по модулю 1 . Эта группа изоморфна $R^{k+s-1} \oplus Z_{2}^{k-1} \oplus T_{1}^{s}$ (соответственно $\left.R^{s-1} \oplus T_{1}^{s}\right)$, где $R$ - аддитивная группа вешественных чисел, $T_{1}$ - аддитивная группа классов вещественных чисел по модулю 1 .

Теорема вытекает из того, что структура дискретной подгруппы группы этого типа имеет вид $Z^{l} \oplus F, 1 \leqslant l \leqslant k+s-1, F-$ коммутативная конечная группа [4, гл. 7, с. 182$]$.

Заметим также, что в случае вещественного спектра $(s=0)$ централизатор $Z(A)$ изоморфен $Z^{l} \oplus Z_{2}^{m}, 1 \leqslant l \leqslant k-1,1 \leqslant m \leqslant k-1$.

Пример 1. Аносовский диффеоморфизм $A: T^{3} \rightarrow T^{3}$ задается матрицей $A=\left(\begin{array}{ccc}0 & 1 & 0 \\ 0 & 0 & 1 \\ -1 & 0 & 3\end{array}\right)$ с характеристическим полиномом $P_{A}(\lambda)=\lambda^{3}-3 \lambda^{2}+1$ и $\operatorname{Spec}(A)=\left(\lambda_{1}, \lambda_{2}, \lambda_{3}\right), \lambda_{1} \in(-1,0)$, $\lambda_{2} \in(0,1), \lambda_{3}=\frac{-1}{\lambda_{1} \lambda_{2}} \in(2,3)$.

Автоморфизм $B=A-I$ принадлежит $Z(A), \operatorname{Spec}(B)=\left(\left(\mu_{1}, \mu_{2}, \mu_{3}\right), \mu_{1}=\lambda_{1}-1 \in\right.$ $\left.(-2,-1), \mu_{2}=\lambda_{2}-1 \in(-1,0), \mu_{3}=\lambda_{3}-1 \in(1,2)\right)$.

Рассмотрим последовательность автоморфизмов $A^{k_{i}} B^{l_{i}}$ такую, чтобы числовая последовательность $\lambda_{2}\left(A^{k_{i}} B^{l_{i}}\right)=\lambda_{1}^{k_{i}} \mu_{1}^{l_{i}}$ имела конечньй предел, отличньй от 0 при $k_{i} \rightarrow+\infty, l_{i} \rightarrow+\infty$. При этом $\lambda_{2}\left(A^{k_{i}} B^{l_{i}}\right)=\lambda_{2}^{k_{i}} \mu_{2}^{l_{i}} \rightarrow 0, \lambda_{3}\left(A^{k_{i}} B^{l_{i}}\right)=\lambda_{3}^{k_{i}} \mu_{3}^{l_{i}} \rightarrow \infty$. Если бы было $Z(A)=Z \oplus Z_{2}$, то $A^{k_{i}} B^{l_{i}}=C^{m_{i}}, C$ - аносовский диффеоморфизм, и ни одна из последовательностей собственных чисел $C^{m_{i}}$ не могла бы иметь конечного предела, отличного от нуля. Таким образом, $Z(A) \cong Z^{2} \oplus Z_{2}$.

Заметим, что в рассмотренном примере $Z(A)$ состоит из аносовских диффеоморфизмов и это всегда так, когда $\operatorname{Spec}(A)$ - вещественньй.

ПримеР 2. Аносовский диффеоморфизм $A: T^{4} \rightarrow T^{4}$ задается матрицей

$$
A=\left(\begin{array}{cccc}
-1 & 1 & 0 & 0 \\
0 & -1 & 1 & 0 \\
0 & 0 & -1 & 1 \\
-1 & 3 & -3 & 2
\end{array}\right)
$$

Введем также в рассмотрение автоморфизм $B=A+I$, его характеристический полином $P_{B}(\lambda)=$ $\lambda^{4}-3 \lambda^{3}+3 \lambda^{2}-3 \lambda+1$ имеет два вещественных корня $\lambda_{1,2}(B)=\frac{3+\sqrt{5}}{4} \pm \frac{\sqrt{6 \sqrt{5}-2}}{4}$ и два комплексных корня $\mu_{1,2}(B)=\frac{3-\sqrt{5}}{4} \pm i \frac{\sqrt{6 \sqrt{5}+2}}{4}, \lambda_{1}(B)>1, \lambda_{2}(B) \in(0,1),\left|\mu_{1}(B)\right|=\left|\mu_{2}(B)\right|=1$. Характеристический полином $A$ имеет следующие корни: $\lambda_{1}(A)=\lambda_{1}(B)-1 \in(0,1), \lambda_{2}(A)=$ $\lambda_{2}(B)-1 \in(-1,0), \mu_{1}(A)=\mu_{1}(B)-1, \mu_{2}(A)=\overline{\mu_{2}(B)}-1,\left|\mu_{1}(A)\right|=\left|\mu_{2}(A)\right|>1$. Автоморфизм $B$, не являюшийся аносовским, принадлежит $Z(A) \cong Z^{2} \oplus F$.

\section{СПИСОК ЛИТЕРАТУРЫ}

[1] Smale S. // Physica D. 1991. V. 5. P. 267-273. [2] Smale S. Mathematical problems for the next century // Preprint, 1997. [3] Касселс Д. В. С. Введение в теорию диофантовых приближений. М.: ИЛ, 1961. [4] Бурбаки Н. Общая топология. М.: Физматгиз, 1959. 